\title{
Insituassessment of the saliva effect on enamel morphology after microabrasion technique
}

\author{
Núbia Inocencya Pavesi Pini ${ }^{1}$, Débora Alves Nunes Leite Lima ${ }^{1}$, Renato Herman Sundfeld ${ }^{2}$, \\ Gláucia Maria Bovi Ambrosano ${ }^{3}$, Flávio Henrique Baggio Aguiar ${ }^{1}$, José Roberto Lovadino ${ }^{1}$
}

${ }^{1}$ Universidade Estadual de Campinas - UNICAMP, Piracicaba Dental School, Department of Restorative Dentistry, Piracicaba, SP, Brazil

Universidade Estadual Paulista - UNESP, Araçatuba Dental School, Department of Restorative Dentistry, Araçatuba, SP, Brazil

${ }^{3}$ Universidade Estadual de Campinas - UNICAMP, Piracicaba Dental School, Department of Social Dentistry, Area of Statistics, Piracicaba, SP, Brazil

\begin{abstract}
Aim: This study evaluated saliva effects on enamel morphology surface after microabrasion technique. Methods: Enamel blocks $\left(16 \mathrm{~mm}^{2}\right)$ obtained from bovine incisors were divided into 9 groups as follows: one control group (no treatment), four groups with microabrasion treatment using $35 \%$ phosphoric acid and pumice $\left(\mathrm{H}_{3} \mathrm{PO}_{4}+\mathrm{Pum}\right)$ and other four groups treated with $6.6 \%$ hydrochloric acid and silica ( $\mathrm{HCl}+\mathrm{Sil})$. One group of each treatment was submitted to 4 frames of saliva exposure: without exposure, 1-h exposure, 24-h exposure, and 7-days exposure on in situ regimen. Nineteen volunteers $(n=19)$, considered as statistical blocks, used an intraoral appliance containing the specimens, for 7 days. Enamel roughness ( $\mathrm{Ra}$ ) was tested before and after treatment, and after saliva exposure. Confocal laser scanning microscopy (CLSM) was used to evaluate qualitatively the enamel morphology. Results: All groups exhibited increased Ra after microabrasion. With regards to saliva exposure, the treatment with $\mathrm{HCl}+\mathrm{Sil}$ presented more susceptibility to the saliva action, but no period of time was efficient in re-establishing this characteristic compared with the control group. CLSM analysis showed reduction of the micro-abrasive damages during the experimental times. Conclusions: Seven days of saliva exposure were not sufficient for the treated enamel to reach its normal characteristics compared with the control group.
\end{abstract}

Keywords: dental enamel; enamel microabrasion; saliva.

\section{Introduction}

The microabrasion technique is a treatment to achieve dental esthetics in cases of superficial tooth stains and enamel decalcification and defects, improving

Received for publication: May 21, 2014 Accepted: August 07, 2014

Correspondence to: Débora Alves Nunes Leite Lima Departamento de Odontologia Restauradora Universidade Estadual de Campinas - Unicamp Faculdade de Odontologia de Piracicaba Avenida Limeira 901 - Caixa Postal 52 CEP: 13414-903, Piracicaba, SP, Brasil Phone: +55 1921015340 E-mail: deboralima@fop.unicamp.br color and surface texture of the tooth ${ }^{1-2}$. It is considered a safe and conservative technique, since it causes a non-significant loss of enamel, and it provides lasting and satisfactory results in a short clinical time ${ }^{1,3-4}$. Currently, the microabrasive agents used are $6.6 \%$ hydrochloric acid with silica, in a commercial presentation, or pumice added to $35 \%$ phosphoric acid, with mechanical application under low-speed rotation ${ }^{5}$. The application of the microabrasive system is commonly followed by enamel polishing with diamond paste pr-7 $^{6-7}$ or fluoridated paste ${ }^{2,4}$.

The clinical success of this technique has been documented ${ }^{1,2,5}$. However, little is known about the effects of the microabrasive systems used on enamel surface, such as roughness and microhardness ${ }^{6-7}$ and its behavior in the oral 
environment in contact with saliva. Since microabrasion provides both erosive and abrasive challenges by an acid, and abrasive and mechanical application, it is expected that the enamel surface will undergo mineral loss due to demineralization, in which saliva and its components have a role in maintaining the integrity of the tissue ${ }^{8-10}$. Surface roughness is important for enamel, since it is related to color, smooth and brittle appearance of the tooth ${ }^{3,11}$.

Compared with in vitro models, in situ and in vivo studies can simulate more closely the clinical conditions ${ }^{8,11}$ and may really reveal influences of microabrasion on enamel. The in situ models are more accessible than the in vivo studies and are considered reliable as they allow the control of some clinical variables ${ }^{8}$, e.g. in relation to the standardization of the enamel surface to be treated with microabrasion and exposed to the oral environment. The literature lacks in situ studies evaluating the treatment of microabrasion and in those cases where in vitro studies are available $e^{3,7,12-13}$ only few of them analyzed the influence of artificial saliva on the enamel after microabrasion ${ }^{6-7}$ and none of them used human saliva. The use of artificial saliva in studies about demineralization creates an optimum environment for the mineral recovery of enamel surface, since it contains only mineral ions in its composition ${ }^{14}$. On the other hand, the treatment of enamel surface with human saliva presents differences in this process, as it contains proteins such as mucin, which is deposited on the surface and may hamper the remineralization process ${ }^{15}$. So, the use of human saliva, in an in situ model, allows the analysis of microabraded enamel, reproducing more reliably the in vivo conditions.

Since there is no study evaluating the behavior of enamel after microabrasion and relating it to human saliva exposure, the present work used an in situ model to investigate the effect of saliva exposure on enamel morphology after different microabrasion techniques.

\section{Material and methods}

The study design is presented in Figure 1.

\section{Volunteers and Ethical Issues}

The study protocol was approved by the local ethics committee in research (Piracicaba Dental School - Protocol No. 037/2011). Nineteen adult volunteers (20-30 years old) met the inclusion criteria exhibiting no fixed or removable orthodontic appliances, good general and oral health, no antibiotic use during 2 months prior to the study, and normal salivary flow. For standardization purposes, the volunteers were instructed to use fluoridated toothpaste (Colgate Total 12, Colgate-Palmolive, São Paulo, SP, Brazil) and manual toothbrushes (Colgate Professional Clean - ColgatePalmolive, São Paulo, SP, Brazil) for 7 days prior to and during the course of the experiment.

\section{Preparation of Specimens}

Enamel slabs ( $4 \times 4 \times 2 \mathrm{~mm})$ were obtained from bovine incisors using a precision saw and a high-concentration diamond disc. To obtain flat and standardized enamel surfaces, the blocks were leveled using water-cooled carborundum discs with increasing grit size in a circular watercooled polishing machine. The resulting surface was then polished with felts associated with a diamond paste of $1,1 / 2$

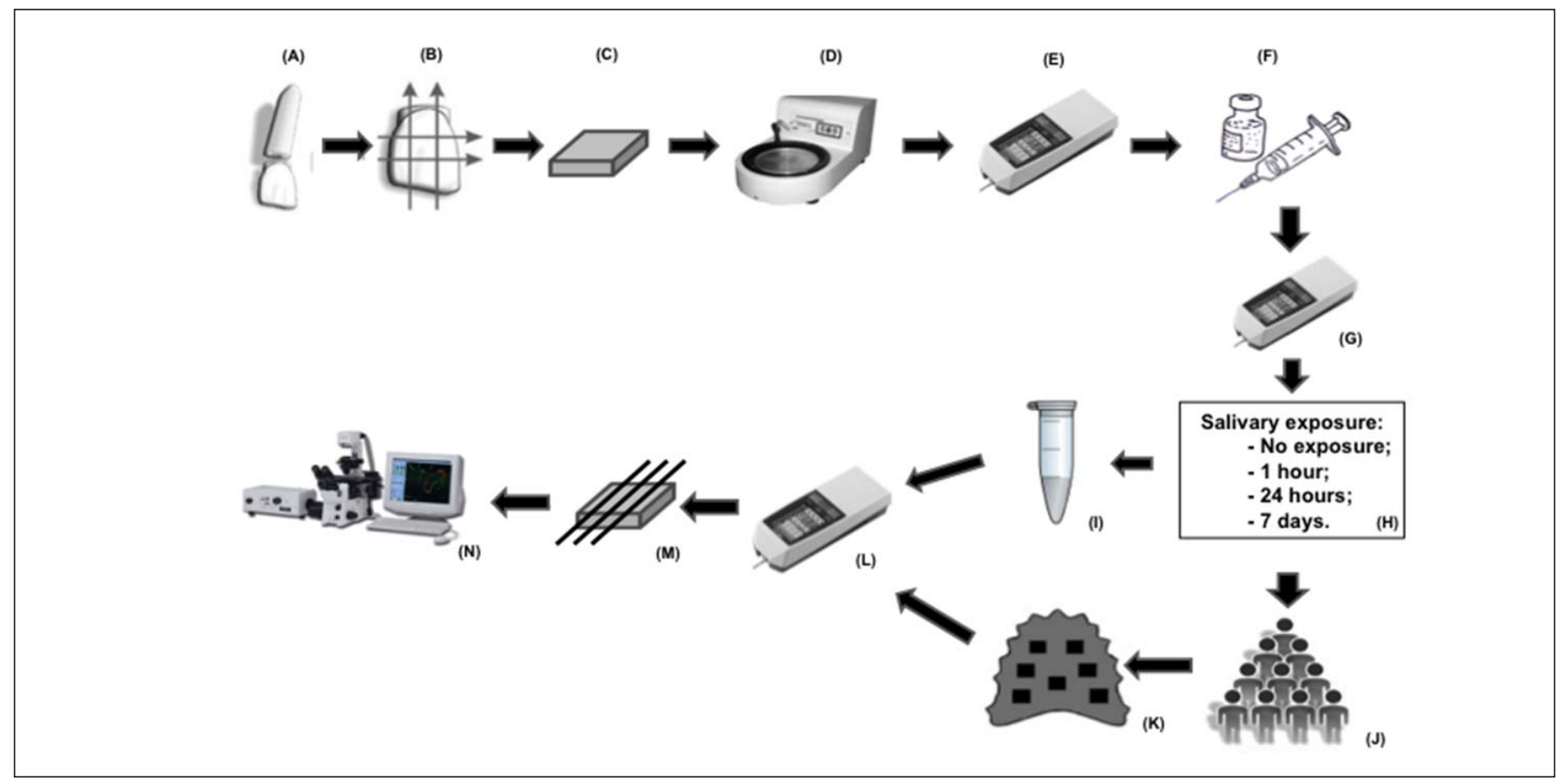

Fig. 1. Experimental design of study (a) Bovine incisor and separation of coronal portion; (b) Sectioning of crowns to obtain specimens; (c) Enamel/dentin blocks with $16 \mathrm{~mm}^{2}$; (d) Polishing of specimens; (e) Roughness analysis; ( $f$ ) Enamel microabrasion; (g) Roughness analysis after treatment; (h) Division of groups according to salivary exposition; (i) Groups without salivary exposition stored in distilled water; (j-k) Groups with salivary exposition distributed for the volunteers; (I) Roughness analysis after salivary exposition; (m) Sectioning of the specimens; (n) Confocal analysis. 
and $1 / 4 \mu \mathrm{m}$ grit sizes and greased with specific oil. Between the polishing steps and after the final polishing, all the slabs were sonicated with distilled water for $15 \mathrm{~min}$. The specimens were sterilized with ethylene oxide.

\section{Groups}

Nineteen volunteers took part in the study and were considered as statistical blocks $(n=19)$, since they used a palatal appliance containing one specimen of each group designed to receive saliva exposure. The samples were divided into nine groups $(\mathrm{n}=19)$ in randomized order and according to the microabrasion technique and storage time in human saliva (in situ phase). The experimental groups were the following: one control group, four groups treated with $\mathrm{H}_{3} \mathrm{PO}_{4}+\mathrm{Pum}$ and four groups treated with $\mathrm{HCl}+\mathrm{Sil}$. The groups with treatment were subdivided according to the in situ regimen: without saliva exposure, $1 \mathrm{~h}, 24 \mathrm{~h}$ and 7 days of saliva exposure.

\section{Surface Treatment}

The enamel microabrasion was performed by $35 \%$ phosphoric acid and pumice $\left(\mathrm{H}_{3} \mathrm{PO}_{4}+\mathrm{Pum}\right)$ or $6.6 \%$ hydrochloric acid and silica $(\mathrm{HCl}+\mathrm{Sil})$. In the first case, equal parts of $35 \%$ phosphoric acid $\left(\mathrm{H}_{3} \mathrm{PO}_{4}\right)$ and pumice were measured with a metering spoon and mixed to compound the microabrasive mixture. Both products were placed on the enamel surface with a syringe until covering the specimen. The microabrasion was achieved with specific rubber cups coupled to an electric micro-motor operating at low speed $(12,000 \mathrm{rpm})$. The treatment regimen was 10 applications of $10 \mathrm{~s}$ each.

\section{Palatal Appliances}

The intra-oral palatal appliances containing seven cavities measuring $4 \times 4 \times 3 \mathrm{~mm}$ (three at each side and one at the center of the appliance) were made for each volunteer in acrylic resin to set the slabs (Figure 1). The enamel slabs were fixed inside the cavities with wax in a manner that the enamel surface was positioned at the same level of the cavity, allowing their contact with the salivary fluid. Each volunteer used its appliance containing seven bovine dental enamel specimens, representing different experimental groups, during an experimental phase of 7 days. When the specimen of the group with 1 hour or 24 hours of salivary exposure was removed from the appliance, the cavity was full of wax. Preferably, the appliances were worn for $24 \mathrm{~h}$, except during meals and periods of oral hygiene. With the appliances in situ, the participants were instructed not to eat, being only allowed to drink water. After meals, a period of at least 15 min elapsed prior to reinsertion of the appliance. In the evening, the appliances, but not the specimens, were cleaned with a toothbrush without toothpaste. Afterwards, the appliances were immersed for $1 \mathrm{~min}$ in chlorhexidine digluconate solution (Periogard; Colgate-Palmolive, São Paulo, SP, Brazil) to avoid plaque formation.

\section{Analysis of the Enamel Roughness}

The enamel roughness $(\mathrm{Ra})$ was analyzed at three time frames: before and after microabrasion, and after saliva exposure using a profilometer roughness tester (Mitutoyo Surfitest 211, São Paulo, SP, Brazil). Three readings were made in three different directions on the enamel surface, and the measurements were averaged.

\section{Confocal Laser Scanning Microscopy (CLSM)}

To visualize enamel surface structures with the confocal laser scanning microscope (Leica TCS SP2-SE Microscopy, Mannheim, Germany), representative specimens of each group were sectioned to obtain slices. Each slice was polished by carbide papers and felts associated with a diamond paste of 1 $\mu \mathrm{m}$ grit size, according to the same protocol described for specimen preparation, resulting in slices about $0.3 \mu \mathrm{m}$ thick. Subsequently, the samples were immersed into a freshly prepared $0.1 \mathrm{mM}$ rhodamine B solution and left for $1 \mathrm{~h}$, without further rinsing ${ }^{16}$. CSLM analysis was performed in fluorescence mode using a 40x magnification objective and oil immersion.

\section{Statistical Analysis}

After an exploratory analysis, the data were analyzed by mixed models for repeated measures (PROC MIXED), followed by Tukey-Kramer and Dunnett tests. Analysis of variance was applied since different treatments were performed in the same sample, at different moments. The significance level was set at $5 \%$.

\section{Results}

All the experimental groups presented increased enamel roughness (Table 1) after microabrasion, with statistically significant differences in comparison with both initial analysis and control group, but without significant differences between them. After saliva exposure, only the group treated with $\mathrm{H}_{3} \mathrm{PO}_{4}+\mathrm{Pum}$ and 1-h exposure showed no decrease in enamel roughness, with statistically significant difference compared with the $R a$ analysis after microabrasion. In the other groups with this treatment, the groups with $24 \mathrm{~h}$ and 7 days of exposure to saliva showed statistical significance in reducing enamel roughness. With regard to the groups treated with $\mathrm{HCl}+\mathrm{Sil}$, all exposure periods to saliva $(1 \mathrm{~h}, 24 \mathrm{~h}$ and 7 days) resulted in decreased enamel roughness compared with the group without saliva exposure. The groups with this treatment and saliva exposure for $24 \mathrm{~h}$ and 7 days to the intraoral environment showed greater reduction of roughness, with statistically significant difference compared with the group without saliva exposure and the group with 1-h saliva exposure. The effect of saliva on enamel roughness for both microabrasive systems showed that groups treated with $\mathrm{H}_{3} \mathrm{PO}_{4}+$ Pum and exposed to saliva for 1 and $24 \mathrm{~h}$ presented the greatest enamel roughness, with statistically significant difference compared with the groups treated with $\mathrm{HCl}+\mathrm{Sil}$ and the group with the same exposure time to the intraoral environment. 
Table 1. Roughness analysis (Ra) means followed by pattern error of all groups.

\begin{tabular}{|c|c|c|c|c|}
\hline \multirow[t]{2}{*}{ Microabrasion } & \multirow[t]{2}{*}{$\begin{array}{l}\text { Salivary } \\
\text { Exposition }\end{array}$} & \multicolumn{3}{|c|}{ Time } \\
\hline & & Before treatment & After treatment & After saliva \\
\hline \multirow{4}{*}{$\mathrm{H}_{3} \mathrm{PO}_{4}+$ Pumice } & No exposure & $0.14(0.03) \mathrm{Ca}$ & ${ }^{\#} 0.36(0.03) \mathrm{Aa}$ & ${ }^{\#} 0.36(0.02) \mathrm{Aa}$ \\
\hline & $1 \mathrm{~h}$ & $0.14(0.03) \mathrm{Ca}$ & ${ }^{\#} 0.41(0.05) \mathrm{Aa}$ & ${ }^{* \#} 0.38(0.02) \mathrm{Aa}$ \\
\hline & $24 \mathrm{~h}$ & $0.13(0.02) \mathrm{Ca}$ & ${ }^{\#} 0.41(0.03) \mathrm{Aa}$ & ${ }^{*} 0.35(0.04) \mathrm{Ba}$ \\
\hline & 7 days & $0.14(0.04) \mathrm{Ca}$ & ${ }^{\#} 0.38(0.03) \mathrm{Aa}$ & ${ }^{\#} 0.21(0.05) \mathrm{Bb}$ \\
\hline \multirow[t]{4}{*}{$\mathrm{HCl}+$ Silica } & No exposure & $0.14(0.03) \mathrm{Ca}$ & ${ }^{\#} 0.39(0.05) \mathrm{Aa}$ & ${ }^{\#} 0.36(0.03) \mathrm{Aa}$ \\
\hline & $1 \mathrm{~h}$ & $0.15(0.03) \mathrm{Ca}$ & ${ }^{\#} 0.41(0.05) \mathrm{Aa}$ & ${ }^{\#} 0.28(0.03) \mathrm{Bb}$ \\
\hline & $24 \mathrm{~h}$ & $0.17(0.04) \mathrm{Ca}$ & ${ }^{\#} 0.38(0.04) \mathrm{Aa}$ & ${ }^{\#} 0.25(0.04) \mathrm{Bbc}$ \\
\hline & 7 days & $0.16(0.02) \mathrm{Ca}$ & ${ }^{\# 0.38}(0.03) \mathrm{Aa}$ & ${ }^{\#} 0.22(0.02) \mathrm{Bc}$ \\
\hline Control & & $0.15(0.03)$ & $0.17(0.03)$ & $0.16(0.02)$ \\
\hline
\end{tabular}

Means followed by different letters (uppercase in horizontal and lowercase in vertical compare time within each category of microabrasion) differ $(\mathrm{p}<0.05)$. *Differ from the group treated with $\mathrm{HCl}+$ Silica with the same time of salivary exposition. \#Differ from the control in the same time of analysis.

The CLSM analysis showed detectable lesions depending on the group, characterized by sites of microwear on enamel surface, for both treatments. During the in situ regimen, there was a tendency towards reduction of this wear by filling of the micro-sites in order to recover the continuous and uniform line of the enamel surface, as found in the control. For both treatments, after 7 days of saliva exposure, almost no microwear sites could be observed and an almost unchanged line characterized the enamel surface (Figure 2).

\section{Discussion}

This study evaluated the action of saliva on the effects of microabrasion techniques, combining an acid, an abrasive agent and mechanical application ${ }^{3,12-13}$, on enamel surface. The clinical protocol proposed by some authors ${ }^{1-2,4-5}$ advises to perform a final polishing of enamel surface after microabrasion. However, this procedure is probably against the purpose of this study, as the polishing reestablishes the enamel roughness ${ }^{6-7}$. As a consequence, it could be stated that if saliva has a positive effect on enamel, it would not be necessary to perform polishing after microabrasion, avoiding additional enamel wear on a demineralized surface.

The results showed changes in enamel roughness, which are considered a function of variations in both surface porosity ${ }^{15-16}$ and mineral $\operatorname{loss}^{15,17}$ resulting from the erosiveabrasive challenge of the microabrasion. In accordance with the results, both microabrasive treatments using $\mathrm{H}_{3} \mathrm{PO}_{4}+\mathrm{Pum}$ or $\mathrm{HCl}+\mathrm{Sil}$ increased the enamel roughness, with statistically significant differences compared with initial analysis and without differences between them. These results are corroborated by previous findings that reported mineral loss and increased enamel porosity after treatment with erosive ${ }^{17}$ and/or abrasive 9-10,12,18-19 treatment and low $\mathrm{pH}^{14}$ compounds.

Enamel microabrasion may be considered an erosiveabrasive model, since the erosive and abrasive challenges cause dissolution of the hydroxyapatite crystals ${ }^{20}$. Although proteins comprise a minor part of the enamel, they have the function of attaching hydroxyapatite crystals together to maintain the hierarchical structure of enamel ${ }^{21}$. Therefore, the effects of microabrasion techniques on the mineral and protein components may include the mechanical behavior of enamel. Both acids showed similar alterations of enamel roughness. This may be related to the concentration of hydrochloric acid at $6.6 \%$, different from those initially proposed for the technique, e.g., $36 \%$ and $18 \% \%^{1,4,22}$, which produce a similar conditioning pattern of $35 \%$ phosphoric acid $^{23}$. With regard to the abrasive, pumice presented excessive abrasiveness and deep erosion compared with the other ${ }^{19}$. As $\mathrm{H}_{3} \mathrm{PO}_{4}+\mathrm{Pum}$ is a microabrasive mixture customized by the clinician, the peculiarities of these components should be considered, as their sensitivity to variations may have clinical outcomes.

As the treated surface became less mineralized, it was possible to observe the saliva buffering capacity during the in situ regimen ${ }^{9,17}$. Fluoride action may be considered even with $1 \mathrm{~h}$ of saliva exposure, since this component is found in oral environment at high concentration up to $10 \mathrm{~h}$ after the last $u^{24} e^{24}$. In this study, the volunteers were instructed to use fluoridated toothpaste because its role should be taken into account. This analysis showed a decrease in roughness, which varied depending on the saliva exposure time in situ regimen and the used microabrasive system. The treatment with $\mathrm{HCl}+\mathrm{Sil}$ could be considered more prone to mineral reposition of saliva than that with $\mathrm{H}_{3} \mathrm{PO}_{4}+$ Pum because, after $1 \mathrm{~h}$ of saliva exposure, it was possible to observe decreased roughness, with statistically significant difference compared with the analysis after microabrasion for this group. For both treatments, 24-h exposure to saliva was efficient in reducing roughness, with statistical differences compared with the analysis after microabrasion. The 7-day exposure time resulted in less roughness than the other exposure times, for both microabrasion treatments. Although the reestablishment of roughness was not observed in the total storage period in saliva applied in this study (7 days), analyzing the progressive results of increase in enamel roughness over time and comparing it with studies that used artificial saliva for 15 days, the authors hypothesized that normal enamel roughness probably would be reached even in a longer evaluation time. This result raises the necessity of performing further studies on this subject. 
Comparing both treatments, the groups treated with $\mathrm{HCl}+\mathrm{Sil}$ presented more evidence of superficial mineral reposition as their results after $1 \mathrm{~h}$ and $24 \mathrm{~h}$ of saliva exposure exhibited the lowest mean values of roughness when compared with $\mathrm{H}_{3} \mathrm{PO}_{4}+\mathrm{Pum}$. The best effects of saliva with the treatment with $\mathrm{HCl}+\mathrm{Sil}$ may be related to specific action of the compounds $\mathrm{HCl}$ and silica. The ion chloride in the buccal environment has been associated with the suppression of hydroxyapatite dissolution ${ }^{25}$ beside the fact that the hydrochloric acid presents lower $\mathrm{pH}$ than the phosphoric acid $^{26}$, which favors surface rehardening and fluoride uptake $^{17,20}$. In relation to silica, it has been used in a bioactive material $\left(\mathrm{Ca}_{3} \mathrm{SiO}_{5}\right)$ as an important agent to induce the remineralization of an acid-etched enamel ${ }^{27}$, since it can provide the link between the calcium from hydroxyapatite and from saliva, leading to the formation of a new apatite layer ${ }^{18,27}$. Therefore, regarding the better results achieved with this treatment, it may be assumed that this compound is formed in the enamel after microabrasion using $\mathrm{HCl}+\mathrm{Sil}$.

None of the treatments was able to re-establish the enamel roughness, as found in control group or in the initial condition, in none of the periods of saliva exposure. In previous in vitro findings, the enamel roughness treated with microabrasion was restored after 15 days of immersion in artificial saliva ${ }^{6-7}$. The differences between these studies ${ }^{6-7}$ and the present one are related to both time of saliva exposure and type of saliva. According to another investigation ${ }^{15}$, the deposition of proteins from saliva (e.g. mucins) on the demineralized substrate may be a factor related to the reduction of re-mineralization. Also, the absence of organic content in artificial saliva may have created an optimized system, thus enhancing the mineral reposition on the eroded surface. To the best of the authors' knowledge, this is the first study that evaluated the effect of the human saliva on the enamel after microabrasion, under in situ conditions. The delay in the reestablishment of the enamel roughness, which did not occur within 7 days, probably is due to the irregular deposition of ions between the enamel prisms, that happen first in the 10 and $25 \mu \mathrm{m}$ layers, possibly due to the topographic characteristics of this region, where Retzius lines have a regular periodicity ${ }^{28-29}$.

Despite of the above information, the in vitro model does not reflect the dynamic conditions of the mouth, such as continuous flow and clearance of saliva, even using artificial saliva ${ }^{29}$. Apart from the similar calcium, phosphate and fluoride ions in artificial saliva, human saliva has some advantages that may account for its superior protective ability, such as formation of an acquired enamel pellicle ${ }^{11}$. This pellicle forms within seconds of exposure to saliva ${ }^{11,14}$ and it has anti-erosive potential because it acts as a diffusion barrier as well as a semi-permeable membrane ${ }^{11,23}$, being important for the enamel re-mineralization. Moreover, saliva action may be enhanced by the presence of re-mineralizing agents containing fluoride ${ }^{8,24}$. With regards to the roughness means obtained with the salivary exposure, it was shown that this property could be possibly restored in a short time. Therefore, it should not be stated that the recovery of mineral content in the enamel after microabrasion is more feasible with artificial saliva, such as in vitro models, since they do not mimic the real circumstances of the oral environment.

The CLSM results showed that the enamel surface had remaining erosive effects from the combined action of acid, abrasive agent and mechanical pressure. The images showed an irregular surface with discontinuity points, characterized by microwear points. The recurrence of these points in the groups with only enamel microabrasion reflects the results on enamel roughness. The CLSM images (Figure 2) for the groups in early stages of saliva exposure (1 and $24 \mathrm{~h}$ ) showed gradual reduction of these points, possibly completing the enamel surface with minerals and ions from saliva. The images also showed that this deposition occurs possibly in a random manner, which could be observed with the roughness results too. After 7 days of saliva exposure in the oral environment, the images demonstrated that the enamel surface tended to be uniform and continuous, as found in the control group, showing the re-mineralizing action of saliva.

A limitation of the present study design should be considered: in clinical situations, enamel microabrasion is performed on the buccal surface of the teeth, whereas in the current study, the enamel blocks were placed on the palate, mainly for the comfort of the volunteers and esthetic implications. Moreover, previous in situ studies ${ }^{8-9,11,24}$ chose the palatal model to simulate the condition of the human

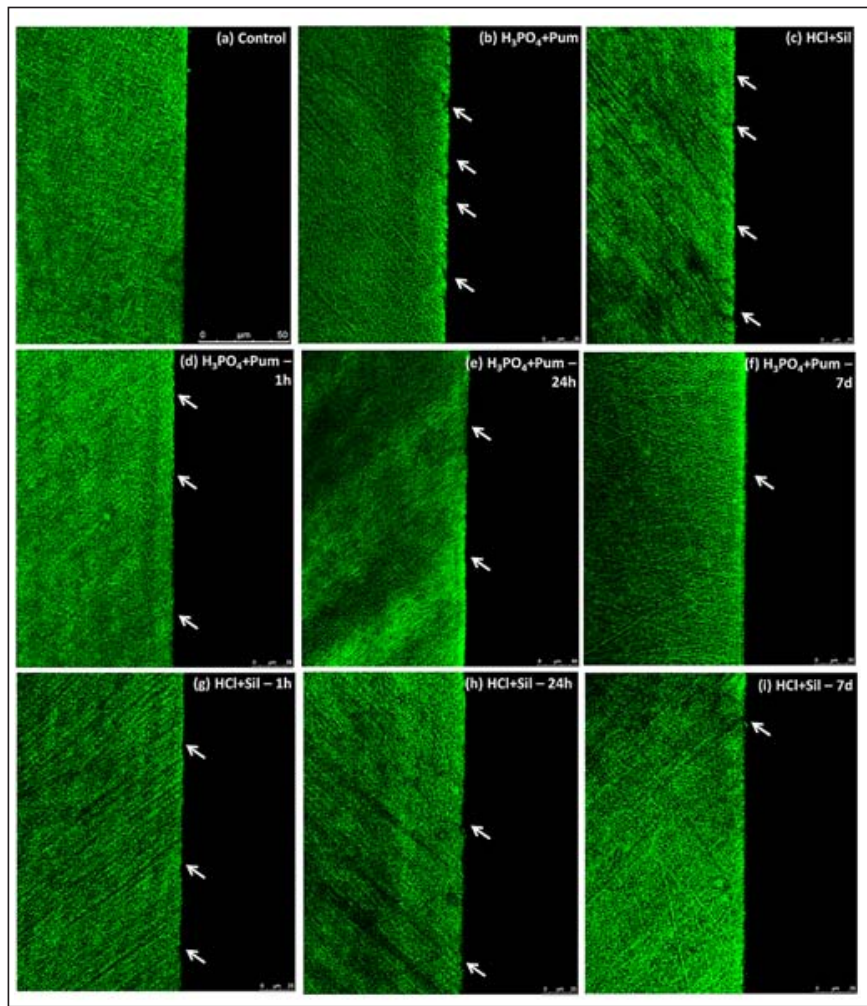

Fig. 2: Representative images of the groups obtained from confocal laser scanning microscopy (CLSM).

Arrows show surface microwear sites on enamel surface resulting from the microabrasion technique and its behavior in the oral environment. (a) Control group; (b) $\mathrm{H}_{3} \mathrm{PO}_{4}+$ Pumice; (c) $\mathrm{HCl}+\mathrm{Sil}$; (d), (e) and (f) $\mathrm{H}_{3} \mathrm{PO}_{4}+$ Pumice with $1 \mathrm{~h}, 24$ $\mathrm{h}$ and 7 days of salivary exposure respectively; (g), (h) and (i) $\mathrm{HCl}+\mathrm{Sil}$ with $1 \mathrm{~h}$, $24 \mathrm{~h}$ and 7 days of salivary exposure respectively. 
oral cavity and successfully proved its superiority over the in vitro strategy.

This is the first in situ study investigating the action of saliva on the enamel morphology subjected to microabrasion treatment. The results showed that the technique causes a significant alteration in enamel roughness, without differences between the tested microabrasive systems. In addition, saliva plays an important role in re-establishing the characteristics of the enamel, since a 7-day exposure to saliva reduced the roughness mean values and the alterations on enamel surface, according to the CLSM analysis.

\section{Acknowledgements}

The authors would like to gratefully acknowledge all the volunteers who participated in this study. This study was supported by Fapesp (Proc. 2011/004067-7).

\section{References}

1. Croll TP. Enamel microabrasion: observations after 10 years. J Am Dent Assoc. 1997; 128: 45S-50S.

2. Sundfeld RH, Rahal V, Croll TP, De Alexandre RS, Briso AL. Enamel microabrasion followed by dental bleaching for patients after orthodontic treatment-case reports. J Esthet and Restor Dent. 2007; 19: 71-7.

3. Meireles SS, Andre D de A, Leida FL, Bocangel JS, Demarco FF. Surface roughness and enamel loss with two microabrasion techniques. J Contemp Dent Pract. 2009; 10: 58-65.

4. Sundfeld RH, Croll TP, Briso AL, de Alexandre RS, Sundfeld-Neto D. Considerations about enamel microabrasion after 18 years. Am J Dent. 2007; 20: 67-72.

5. Wang Y, Sa Y, Liang S, Jiang T. Minimally Invasive Treatment for Esthetic Management of Severe Dental Fluorosis: A Case Report. Oper Dent. 2012; 38: 358-62.

6. Bertoldo CES, Pini NIP, Miranda DA, Catelan A, Ambrosano GMB, Lima DANL, et al. Physicochemical properties of enamel after microabrasion technique. J Res Dent. 2014; 2: 176-88.

7. Fragoso LS, Lima DA, de Alexandre RS, Bertoldo CE, Aguiar FH, Lovadino JR. Evaluation of physical properties of enamel after microabrasion, polishing, and storage in artificial saliva. Biomed Mater. 2011; 6: 035001.

8. Calvo AF, Tabchy CP, Del Bel Cury AA, Tenuta LM, da Silva WJ, Cury JA. Effect of acidulated phosphate fluoride gel application time on enamel demineralization of deciduous and permanentteeth. Caries Res. 2012; 46:31-7.

9. Grazziotin GB, Rios D, Honorio HM, Silva SM, Lima JE. In situ investigation of the remineralizing effect of saliva and fluoride on enamel following prophylaxis using sodium bicarbonate. Eur J Dent. 2011; 5: 40-6.

10. Ribeiro HZ, Lima JE, Vono BG, Machado MA, da Silva SM. Airpolishing effect on bovine enamel and the posterior remineralizing effect of saliva. An in vitro study. J Appl Oral Sci. 2006; 14: 193-7.

11. Sa Y, Sun L, Wang Z, Ma X, Liang S, Xing W, et al. Effects of two in-office bleaching agents with different $\mathrm{pH}$ on the structure of human enamel: an in situ and in vitro study. Oper Dent. 2013; 38: 100-10.

12. Paic M, Sener B, Schug J, Schmidlin PR. Effects of microabrasion on substance loss, surface roughness, and colorimetric changes on enamel in vitro. Quint Int. 2008; 39: 517-22.

13. Schmidlin PR, Gohring TN, Schug J, Lutz F. Histological, morphological, profilometric and optical changes of human tooth enamel after microabrasion. Am J Dent. 2003; 16: 4A-8A.

14. Siqueira WL, Custodio W, McDonald EE. New insights into the composition and functions of the acquired enamel pellicle. J Dent Res. 2012; 91: 1110-8.
15. Hara AT, Gonzalez-Cabezas C, Creeth J, Zero DT. The effect of human saliva substitutes in an erosion-abrasion cycling model. Eur J Oral Sci. 2008; 116: 552-6.

16. De Abreu DA, Sasaki RT, Amaral FL, Florio FM, Basting RT. Effect of home-use and in-office bleaching agents containing hydrogen peroxide associated with amorphous calcium phosphate on enamel microhardness and surface roughness. J Esthet Restor Dent. 2011; 23: 158-68.

17. Gonzalez-Cabezas $\mathrm{C}$, Jiang $\mathrm{H}$, Fontana M, Eckert $\mathrm{G}$. Effect of low pH on surface rehardening efficacy of high concentration fluoride treatments on non-cavitated lesions. J Dent. 2012; 40: 522-6.

18. Dong ZC J, Deng $Y$, Joiner $A$. In vitro remineralization of acid-etched human enamel with Ca3SiO5. App Surf Sci. 2010; 256: 3.

19. Honorio HM, Rios D, Abdo RC, Machado MA. Effect of different prophylaxis methods on sound and demineralized enamel. J App Oral Sci. 2006; 14: 117-23.

20. Buzalaf MA, Hannas AR, Magalhaes AC, Rios D, Honorio HM, Delbem AC. pH-cycling models for in vitro evaluation of the efficacy of fluoridated dentifrices for caries control: strengths and limitations. J App Oral Sci. 2010; 18: 316-34.

21. Dominguez JA, Bittencourt B, Michel M, Sabino N, Gomes JC, Gomes OM. Ultrastructural evaluation of enamel after dental bleaching associated with fluoride. Microsc Res Tec. 2012; 75: 1093-8.

22. Dalzell DP, Howes RI, Hubler PM. Microabrasion: effect of time, number of applications, and pressure on enamel loss. Ped Dent. 1995; 17: 207-11.

23. Wiegand A, Bliggenstorfer S, Magalhaes AC, Sener B, Attin T. Impact of the in situ formed salivary pellicle on enamel and dentine erosion induced by different acids. Acta Odontol Scand. 2008; 66: 225-30.

24. Paes Leme AF, Dalcico R, Tabchy CP, Del Bel Cury AA, Rosalen PL, Cury JA. In situ effect of frequent sucrose exposure on enamel demineralization and on plaque composition after APF application and $F$ dentifrice use. J Dent Res. 2004; 83: 71-5.

25. Jager $D H$, Vieira AM, Ligtenberg AJ, Bronkhorst $E$, Huysmans MC VissinkA. Effect of salivary factors on the susceptibility of hydroxyapatite to early erosion. Caries Res. 2011; 45: 532-7.

26. Meyer-Lueckel H, Paris S, Kielbassa AM. Surface layer erosion of natural caries lesions with phosphoric and hydrochloric acid gels in preparation for resin infiltration. Caries Res. 2007; 41: 223-30.

27. Wang Y, Li X, Chang J, Wu C, Deng Y. Effect of tricalcium silicate (Ca3SiO5) bioactive material on reducing enamel demineralization: An in vitro pH-cycling study. J Dent. 2012; 40: 1119-26.

28. Torres-Rodriguez C, Gonzalez-Lopez S, Bolanos-Carmona V, SanchezSanchez P, Rodriguez-Navarro A, Attin T. Demineralization effects of phosphoric acid on surface and subsurface bovine enamel bleached with in-office hydrogen peroxide. JAdhes Dent. 2011; 13: 315-21.

29. Li C, Risnes S. SEM observations of Retzius lines and prism crossstriations in human dental enamel after different acid etching regimes. Arch Oral Biol. 2004; 49: 45-52. 